\title{
Descriptions and depictions of environments
}

\author{
HOLLY A. TAYLOR and BARBARA TVERSKY \\ Stanford University, Stanford, California
}

\begin{abstract}
Subjects studied maps with the expectation that they would draw or describe them from memory. In fact, subjects did both. Order of drawing or describing landmarks revealed the mental organization of environments. Organization was quite similar across maps and descriptions of the same environments, revealing hierarchical structures based on spatial and functional features of the environments and on conventions for sequencing the landmarks.
\end{abstract}

Before there was written language, there were pictures: cave drawings, petroglyphs, maps, tallies, and picture languages (Gelb, 1963). These various communicative inventions of the human mind are remarkably similar across cultures that have had no known contact. The resemblance of the petroglyphs left by North American Indians to those of other cultures was enough to convince early observers that America had been invaded by Chinese, or Egyptians, or the Ten Lost Tribes (Gelb, 1963). The rock paintings of the Bushmen in South Africa create depth in much the same way as those left in caves in southern France and northern Spain (Boas, 1927/1955). The geometric patterns in weavings in basketry from many different parts of the world resemble each other, though their symbolic interpretations may differ (Boas, 1927/1955).

Not only do the depictions and designs of unrelated cultures resemble each other, they also resemble the spontaneous productions of children. Children's drawings spontaneously use, albeit crudely, many of the conventions of representing perspective used by artists in various cultures (Hagen, 1986). The maps of early explorers and even modern tourist maps bear similarities to the maps of children and adults (for examples, see Brown, 1949/1977; Noble, 1981; Southworth \& Southworth, 1982). Unlike "veridical" maps, they often incorporate several scales and several perspectives. Like veridical maps, they often include symbolic elements as well as the strictly visual.

The presence of widespread similarities in pictorial representations and their underlying systematicity suggests that they reflect universal cognitive predilections, in conjunction with constraints of the media. Despite these compelling similarities, not just of form, but of meaning, pictorial productions are rarely used as psychological data.

One of the hesitations in using pictures as cognitive data is that adults rarely draw them, so that their naturalness

This research was sponsored by the Air Force Office of Scientific Research, Air Force Systems Command, USAF, under Grant AFOSR 89-0076. H.A.T. is supported by an AFOSR LGFP predoctoral fellowship. We are grateful to Herb Clark for discussions of descriptions and to Christa Hansen for discussions of depictions. Correspondence should be addressed to H. A. Taylor, Department of Psychology, Building 420, Stanford University, Stanford, California 94305-2130. or ecological validity as data may have questionable. However, one situation in which adults quite commonly draw sketches is when they draw maps to communicate spatial information. Depictions of maps served as one of the dependent variables of the present experiment. Constrained map productions have been used as data, primarily to indicate systematic distortions (e.g., Chase \& Chi, 1981; Stevens \& Coupe, 1978; Tversky, 1981; Tversky \& Schiano, 1989). Another difficulty in using pictures as data is scoring them. In this case, depictions were scored for organization, with the index of organization being the order in which the elements of the map were drawn. Order of output has been used as an index of organization at least since Tulving (1962) used order of free recall of unrelated words as an index of clustering or chunking. The assumption underlying that work, and the many subsequent techniques developed, is that items that are more related are more likely to be remembered together, an assumption basic to all conceptions of associative memory.

In addition to depictions of maps, descriptions of maps served as a dependent variable. Descriptions can also be scored for organization on the basis of recall order of the landmarks. Therefore, there is an added convenience that the same measure was used for both drawings and descriptions. Subjects learned one of three environments, which varied in scale. Two of these environments were adapted from previous research (Taylor \& Tversky, 1992), a small-scale Convention Center and a large-scale Town, and one environment, an Amusement Park midway in scale, was new. These were meant to be representative of the sorts of environments that people spontaneously draw map sketches of. Subjects studied a map with the expectation that they would either draw the map or describe the environment. In actuality, they performed both tasks, in counterbalanced order. Our interest was in how people organized these spatial environments. Several factors could influence organization, including (1) expectations, to draw or to describe; (2) task constraints, for either drawing or describing; and (3) characteristics of the environments. In the last case, similarities will emerge between the organization revealed by the descriptions and that revealed by the depictions.

There are several reasons to expect that organization of depictions may differ from that of descriptions. For 
one thing, there may be constraints imposed by the media themselves. Discourse, it has been observed (e.g., Levelt, 1982), is linear. Of course, pictures must be drawn sequentially, too; but in discourse the order must make sense to both sender and receiver as the discourse progresses. A drawing does not need to be comprehended until it is completed, so that the order in which elements are drawn is not part of the communicative act. There are other factors that may affect drawing. First, it may be easier to draw nearby elements together, a principle of least effort or manual economy. Next, drawing is known to be affected by writing habits (e.g., Goodnow, 1977; Kugelmass \& Lieblich, 1979; Tversky, Kugelmass, \& Winter, 1991), so the left-right, top-bottom directions governing written English may be evident in the drawings of English speakers. On the other hand, the order for both discourse and drawing may be driven more by expectations subjects have about the task they will perform. These expectations might be influenced by issues such as constraints of the media, linearization, manual economy, or writing habits.

There are reasons to expect that the anticipated task either drawing or describing, may lead to differential learning and therefore differential organization of the original map. The exact spatial relations among elements are more critical for maps than for descriptions. Similarly, the translation of the spatial to the verbal and the sequencing of elements are more critical for describing than for drawing. The task of constructing a description from a depiction seems to require more transformations than does the task of constructing a depiction from a depiction. In previous work, learners encoded the same pictorial stimuli quite differently in anticipation of picture recognition than in anticipation of verbal recall (Tversky, 1973). Learners may attempt to form a mental image of the map when expecting to draw a map and may attempt to form an implicit description of the map when expecting to describe it. On the other hand, aspects of the natural spatial structure of the environments may determine the organization and override considerations of anticipated response mode.

There is evidence from many studies using a variety of memory measures that environments, like linguistic stimuli, are grouped and organized in memory (e.g., Chase \& Chi, 1981; Hirtle \& Jonides, 1985; McNamara, 1986; Stevens \& Coupe, 1978; Tversky, 1992). Spatial and visual features, as well as semantic ones, serve as bases for organization. In mental representations of maps, larger regions can be divided into smaller ones. Map landmarks are often remembered with respect to the smaller regions encompassing them, and the smaller regions are remembered relative to the larger ones. This is a hierarchy based on containment or part-whole relations and may lead to systematic distortions in direction estimates (Stevens \& Coupe, 1978). Regional boundaries, significant highways, rivers, and other salient natural features of environments may be used to subdivide environments. More salient environmental features may have precedence over less salient ones. Nonspatial organizations may also be used-for example, remembering items together that are related by function rather than by spatial proximity. In research on students' mental representations of Ann Arbor, Hirtle and Jonides found that commercial buildings tended to be grouped with other commercial buildings, and university buildings with other university buildings, despite the fact that the buildings were spatially interspersed. The groupings affected distance estimates, with between-group estimates overestimated relative to within-group estimates.

\section{METHOD}

\section{Subjects}

Seventy undergraduates from Stanford University participated individually, either for pay or in partial fulfillment of a course requirement for introductory psychology. Approximately equal numbers of male and female subjects were recruited. All subjects recruited were native English speakers. The data from 3 subjects, 2 subjects who turned out to be non-native English speakers and 1 subject who did not follow instructions, were eliminated from analysis.

\section{Materials}

Three fictitious environments were drawn using an Apple Macintosh and the software MacPaint and were printed on standard 8.5 $\times 11$ in. paper. The environments differed in scale, ranging from a single building to an enclosed Amusement Park with several buildings to a small Town. The building, a Convention Center, contained 13 landmarks. The Amusement Park contained 17 landmarks, and the Town contained 13 landmarks. The Town and the Convention Center were adapted from previous research (Taylor \& Tversky, 1992). The maps appear in Figures 1-3. A compass rose appeared on each map, indicating that the maps were oriented with North at the top.

\section{Design and Procedure}

The subjects received one of two instruction sheets. Both instructions informed the subjects that they would study a map for 5 min in order to later recall the information. The instructions described different recall tasks, one telling the subjects they would draw the map from memory and the other telling the subjects they would write a verbal description of the map. The instructions for the description told the subjects to write their description so that someone who was unfamiliar with the environment and had never seen the map could read the description and know where all the landmarks were. The experimenter then clarified any questions about the procedure.

The subjects received one of the three maps to study. Overall, 24 subjects received the map of the Town, 24 received the Convention Center map, and 22 received the Amusement Park map. Of the subjects eliminated from analysis, 2 received the Town map and 1 received the Convention Center map. The subjects could study the map for as long as $5 \mathrm{~min}$, but they could move on to the recall task whenever ready. After the study phase, the experimenter told the subjects that instead of the single task described on the instruction sheet, they would actually be asked to do two memory tasks, draw a map and write a description. Order of tasks was counterbalanced across subjects so that half the subjects performed the ex- 


\section{TOWN}

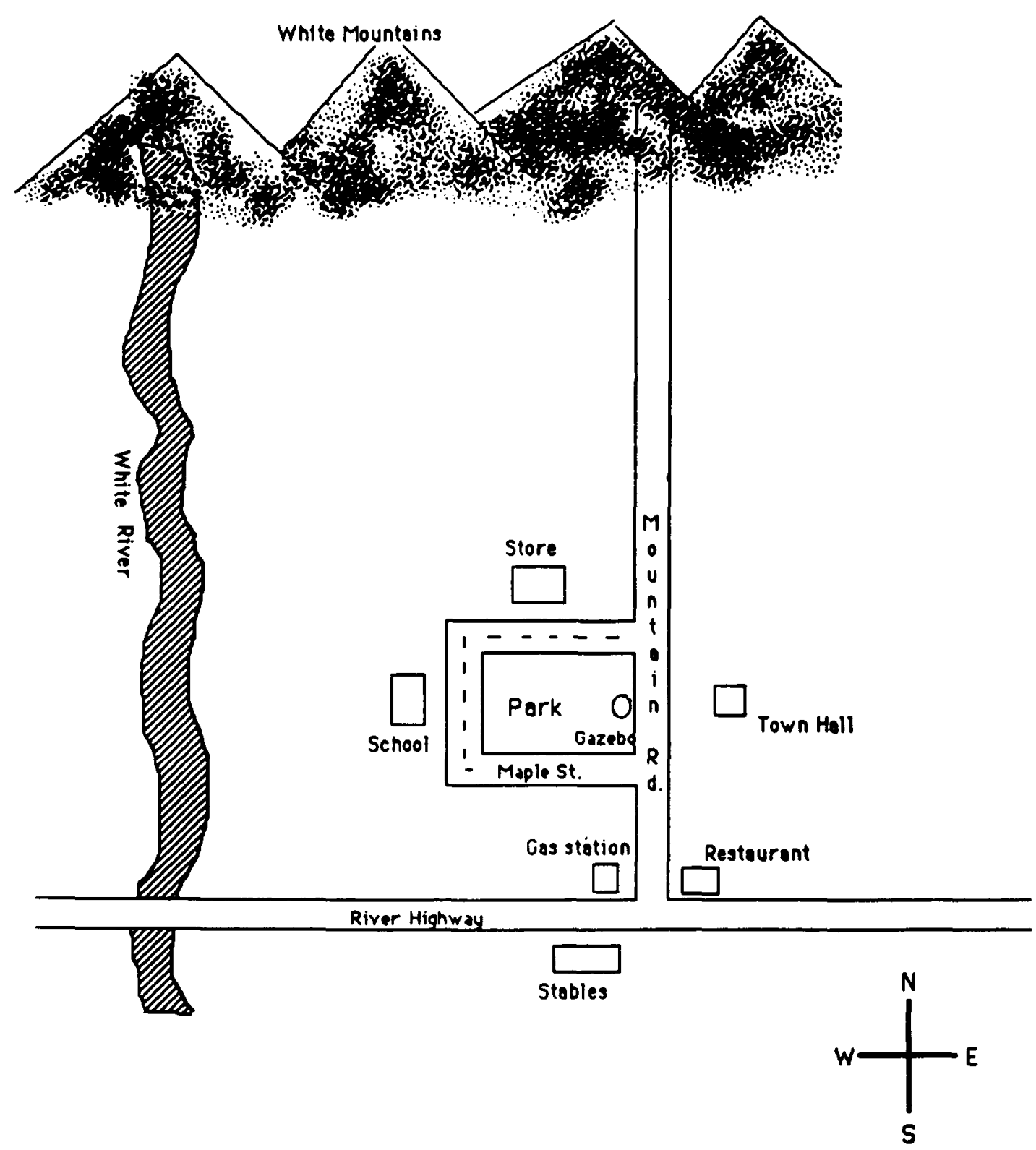

Figure 1. Map of Town. Adapted from Taylor and Tversky (1992) with permission.

pected task first and half performed the surprise task first. While subjects drew their maps, the experimenter recorded the order in which each item was drawn. The subjects completed the tasks at their own pace, but within $30 \mathrm{~min}$.

\section{RESULTS}

\section{Memory Results}

Landmarks. Overall, the subjects' memory was excellent. Table 1 shows the total number of landmarks in each environment and the mean number of landmarks re- called on each memory task. Neither the task expectation, the first task performed, nor the match between these variables affected memory. For maps and descriptions combined, the subjects recalled $94.6 \%$ of the landmarks. Although performance on both memory tasks was high, a paired $t$ test showed that the subjects remembered significantly more landmarks when drawing maps $(\mathbf{9 8 . 2 \%})$ than when writing descriptions $(94.6 \%)[t(66)=3.58, p<$ $.001]$. The memory difference between maps and descriptions held up, with at least marginal significance, regardless of the expected task or the first task performed, for 


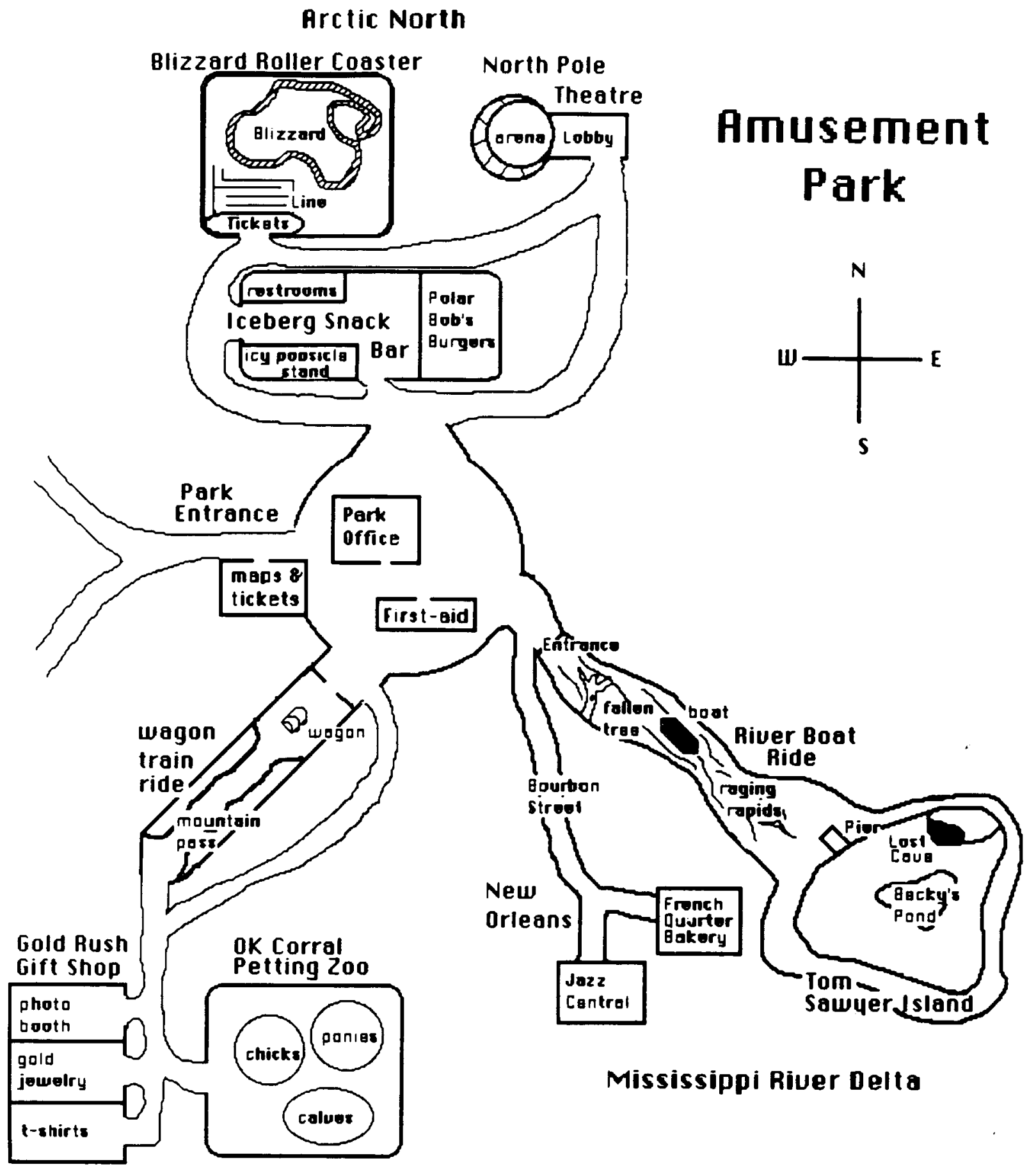

Wild Wild Wes t

Figure 2. Map of Amusement Park. 


\section{CONVENTION CENTER}
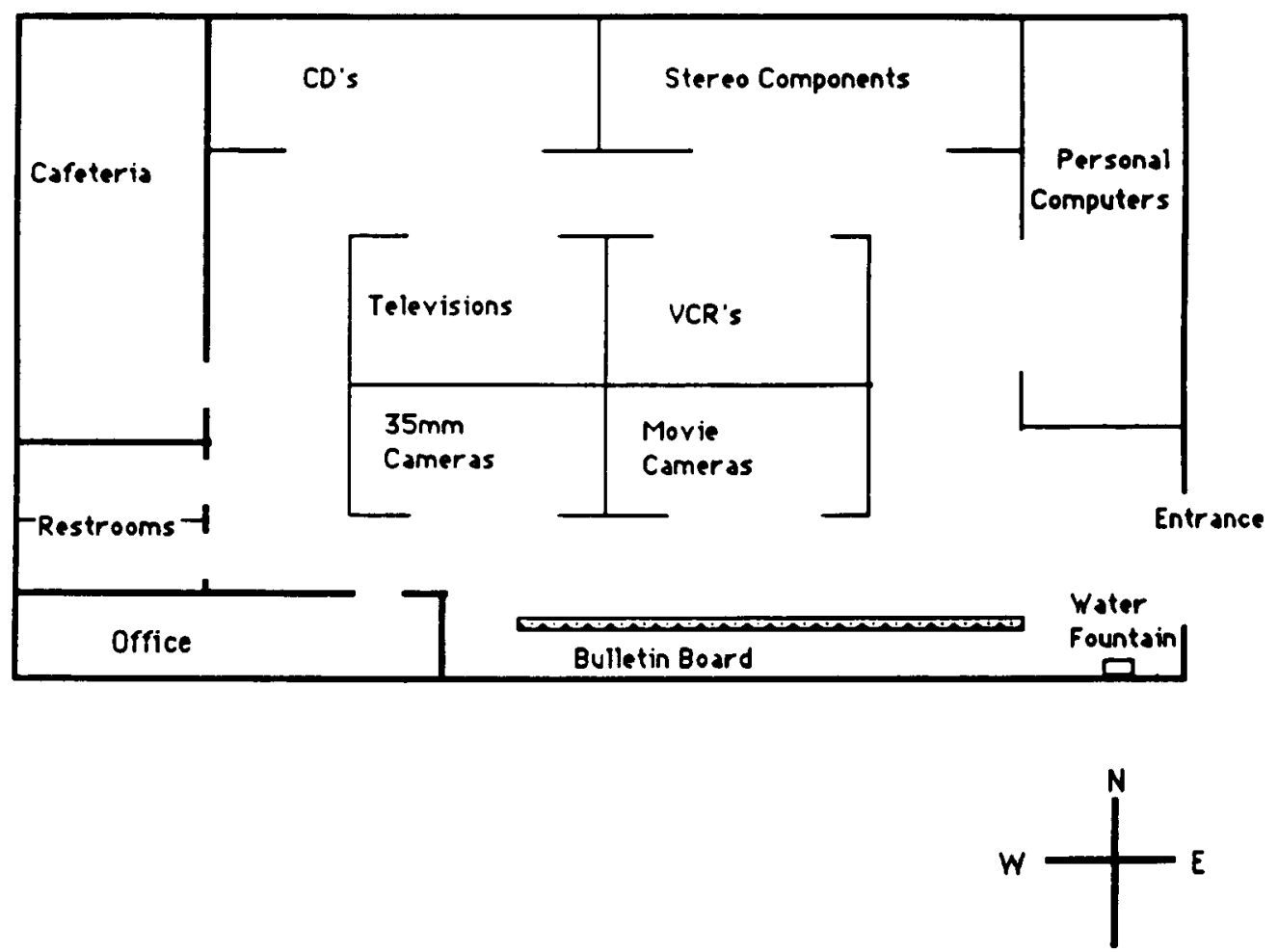

S

Figure 3. Map of Convention Center. Adapted from Taylor and Tversky (1992) with permission.

subjects expecting to draw a map $[t(32)=3.09$, $p<.01]$, for those expecting to write a description $[t(33)=1.96, p<.1]$, for those who first drew the map $[t(32)=2.08, p<.05]$, and for those who first wrote a description $[t(33)=2.95, p<.01]$. Examples of descriptions of the three environments are shown in the Appendix.

Spatial information: Maps. Inclusion of landmarks in both descriptions and depictions was only one part of the task. The other part was to accurately convey the spatial information relating the landmarks to one another. The maps the subjects drew were scored for omissions and for location errors. There were three types of location errors: global errors, local errors, and indeterminate locations. Global errors occurred when entire sections were misplaced but spatial relations between landmarks within a section were correct. Local errors occurred when individual landmarks were misplaced within their section. Indeterminate location errors occurred when the subjects provided a label for a landmark but did not provide an icon signifying the location. On average, the subjects made fewer than one error of any type per map, so that errors were not broken down by type. Error rates did not differ significantly for the individual environments.

Spatial information: Descriptions. The descriptions made obvious the fact that the subjects treated the maps as environments and not simply as spatial arrays. Therefore, the subjects followed the description instructions by conveying the spatial layout of the environment to an unknown reader. Determining whether the descriptions accurately conveyed spatial information was more difficult. To do so, we recruited a new group of subjects. We gave each description to 2 subjects who had never seen the associated map. They were asked to construct a map from the description. Errors on these maps could come from two sources: errors and ambiguities in the descriptions, or misinterpretations and drawing errors by the new subjects. Because we were interested in how well the descriptions could communicate, we scored the map with the lower number of errors for each description. These maps were scored as described above. On average, the subjects included $90.8 \%$ of landmarks in their maps, compared with $94.6 \%$ of landmarks included in the descrip-

Table 1

Mean Landmarks Recalled

\begin{tabular}{lccc}
\hline & & \multicolumn{2}{c}{ Memory Task } \\
\cline { 3 - 4 } & $N$ & Map & Description \\
\hline Town & 13 & 12.7 & 11.9 \\
Amusement Park & 17 & 16.8 & 16.5 \\
Convention Center & 13 & 12.7 & 12.4 \\
\hline
\end{tabular}


tions. The subjects included $91.3 \%$ of the landmarks for the Town map and $\mathbf{9 0 . 5 \%}$ for both the Amusement Park and the Convention Center. On each map, it was possible to have more location errors than locations, since there were different types of location errors. However, the subjects made relatively few location errors. Overall, the subjects made very few global errors; there were 0.28 global errors on average, with 0.23 errors on the Town map, 0.24 errors on the Amusement Park map, and 0.36 errors on the Convention Center map. They also did not leave many locations indeterminate; there were 0.35 indeterminate locations on average, with 0.18 for the Town map, 0.29 for the Amusement Park map, and 0.56 for the Convention Center map. There were more local errors, 1.51 local errors on average, with 1.32 errors for the Town map, 1.81 errors for the Amusement Park map, and 1.44 errors for the Convention Center map. These low error rates show that the descriptions conveyed spatial information quite accurately.

\section{Order Results}

For each subject, recall order on both memory tasks was listed. In descriptions, recall order was based on first mention of a landmark. In drawings, order was based on placement of an icon representing a landmark rather than on the order that the items were labeled. Comparing recall order both within and across subjects and tasks required the same map elements. However, the subjects sometimes forgot landmarks in one or both of the memory tasks. To yield the greatest number of usable recall orders for analysis, we determined the landmarks most often forgotten for each environment and eliminated those from the lists. In all, 3 of 13 landmarks were eliminated from the Town orderings, 2 of 17 from the Amusement Park orderings, and 3 of 13 from the Convention Center orderings. Recall orders that still had missing landmarks were eliminated from the analysis. The analyses used these remaining recall orders to examine three issues: (1) the degree of agreement on recall order across subjects on the two tasks, (2) the degree of agreement on recall order within subjects on the two tasks, and (3) tendencies for landmarks to cluster together in recall.

Order agreement across subjects. To determine the degree of agreement across subjects for each memory task, we calculated Kendall's coefficient of concordance across subjects for the map orders, the description orders, and the orders of the two tasks together. Kendall's coefficient of concordance shows the extent to which distinct rank orderings are similar. With a sizable number of sub- jects, significance can be tested using a chi-square distribution. For all three environments, analysis of each task separately and both tasks combined yielded significant agreement. This point will be addressed further in the next section. Table 2 displays Kendall's coefficients of concordance by environment and task. All chi-squares were significant. For the Town, map-drawing agreement yielded $\chi^{2}(9, N=20)=145.30, p<.001$; descriptionorder agreement yielded $\chi^{2}(9, N=17)=90.85, p<.001$; and combined-task-order agreement yielded $\chi^{2}(9, N=37)$ $=229.37, p<.001$. For the Amusement Park, mapdrawing agreement yielded $\chi^{2}(14, N=21)=95.02$, $p<.001$; description-order agreement yielded $\chi^{2}(14, N=20)=184.77, p<.001$; and combined-taskorder agreement yielded $\chi^{2}(14, N=41)=225.93$, $p<.001$. For the Convention Center, map-drawing agreement yielded $\chi^{2}(9, N=22)=75.08, p<.001$; description-order agreement yielded $\chi^{2}(9, N=20)=$ 85.54, $p<.001$; and combined-task-order agreement yielded $\chi^{2}(9, N=42)=156.64, p<.001$.

We also calculated agreement of orders depending on the task expected and on the first task performed. Both expectations and both first tasks resulted in significant agreement for all environments. The chi-square values showing significant concordance for these four conditions ranged between 71.65 and 157.11 , with degrees of freedom equal to the number of landmarks in the environment minus one, and $p s<.01$. Expecting a description seemed to lead to greater concordance than expecting a map, for all three environments. To test this hypothesis, we calculated the average $r$ value associated with each coefficient of concordance. This resulted in six $r$ values, one for each expectation in each of the three environments. For each of these, we calculated the Fisher's $r$-to- $z$ transformation, which resulted in six $z$ scores. A paired $t$ test on these values did not result in a significant difference in concordance. However, since the coefficient of concordance is a summary statistic, little power was left for comparison of the expectations. Overall, expectation of a map resulted in an average $z$ score of the concordance coefficient of 0.45 , and expectation of a description resulted in an average $z$ score of 0.66 . The task performed first did not influence the degree of agreement.

Order agreement within subjects between tasks. As shown in the previous section, when the recall order for both tasks was combined across subjects, the results showed significant agreement on order. This combined measure indicates that the subjects tended to recall landmarks in the same order for both tasks. To further

Table 2

Order Agreement Across and Within Subjects

\begin{tabular}{lcccc}
\hline & \multicolumn{3}{c}{ Kendall's Coefficient of Concordance } & Tau: Map and \\
\cline { 2 - 5 } & Maps & Descriptions & Both & Description \\
\hline Town & .81 & .59 & .69 & .68 \\
Amusement Park & .32 & .66 & .39 & .48 \\
Convention Center & .38 & .48 & .41 & .37 \\
\hline
\end{tabular}


determine the degree of agreement between tasks, we calculated Kendall's tau for the two memory-task recall orders for each subject and then averaged these for each environment. Kendall's tau indicates the agreement between two rank orderings by computing the number of order inversions for pairs of items in the two rankings. The average taus were $0.63,0.48$, and 0.37 for the Town, Amusement Park, and Convention Center, respectively. For comparison with the concordance coefficients, the tau values are also displayed in Table 2 . For $\mathbf{1 0}$ or more items, the significance of Kendall's tau can be tested using a normal distribution. The subjects showed highly significant agreement between tasks for both the Town $(z=$ $2.54, p<01)$ and the Amusement Park $(z=2.49$, $p<.01)$ and marginally significant agreement for the Convention Center $(z=1.49, p<.10)$.

Overall, the correspondences within and across tasks and within and across subjects were strong but variable and by no means perfect. One reason for the imperfect correspondences was that each environment, especially the Amusement Park and the Convention Center, elicited more than one popular order. For example, for the Amusement Park, some subjects began at the entrance and others at the northern section. For the Convention Center, some subjects first recalled the outer core of exhibits and then the inner core, whereas others alternated between outer and inner corridors. The alternatives for each environment will be documented in the section on specific features of orders.

Clustering of landmarks. To better understand the bases of the strong recall-order concordance, the next step involved a closer look at the orders by examining the clustering of landmarks in recall. For each subject, we calculated the recall distance for every pairwise combination of landmarks in the environment, specifically, the number of landmarks recalled between the two items in the pair. Note that distance depends on recall order and not on map distance. From each recall order, the average recall distance for each pair of landmarks was calculated and represented in a half matrix. This was done separately for each environment and for descriptions and maps. The average distance matrix was then used as input for ADDTREE (Sattath \& Tversky, 1977). This program produces a tree, with landmarks represented by the external nodes, where the distance between landmarks is given by the length of the horizontal part of the path that joins them (the vertical part is added for graphic convenience). ADDTREE is less restrictive than the standard hierarchical clustering scheme, which requires that all external nodes be equidistant from the root.

The ADDTREE solutions yielded interpretable representations. These solutions accounted for $93.6 \%$ of the variance for descriptions and $93.1 \%$ of the variance for maps. The fit of the ADDTREE solutions did not differ for the two memory tasks or for the different environments. Figures 4-9 show the ADDTREE solution trees of the three environments.

A visual comparison of ADDTREE solutions obtained for maps and descriptions reveals that the maps yielded more clusters, whereas the descriptions are more chainlike. For example, in the Amusement Park, the map solution gave rise to three distinct clusters corresponding to the entrance, the North, and the South, whereas the description solution gave a chain of landmarks. Despite these apparent detailed differences between solutions for maps and descriptions, in each case the larger groupings tended to contain the same set of landmarks. For the Town, the large-scale environmental features constituted one cluster, the landmarks at the intersection constituted a second cluster, and the landmarks around the square constituted a third cluster. For the Amusement Park, the clusters were geographic and functional. For the Convention Center, the groupings corresponded to the walls of the Center and the inner core. These similarities of structure are consis-

Town: Description Order

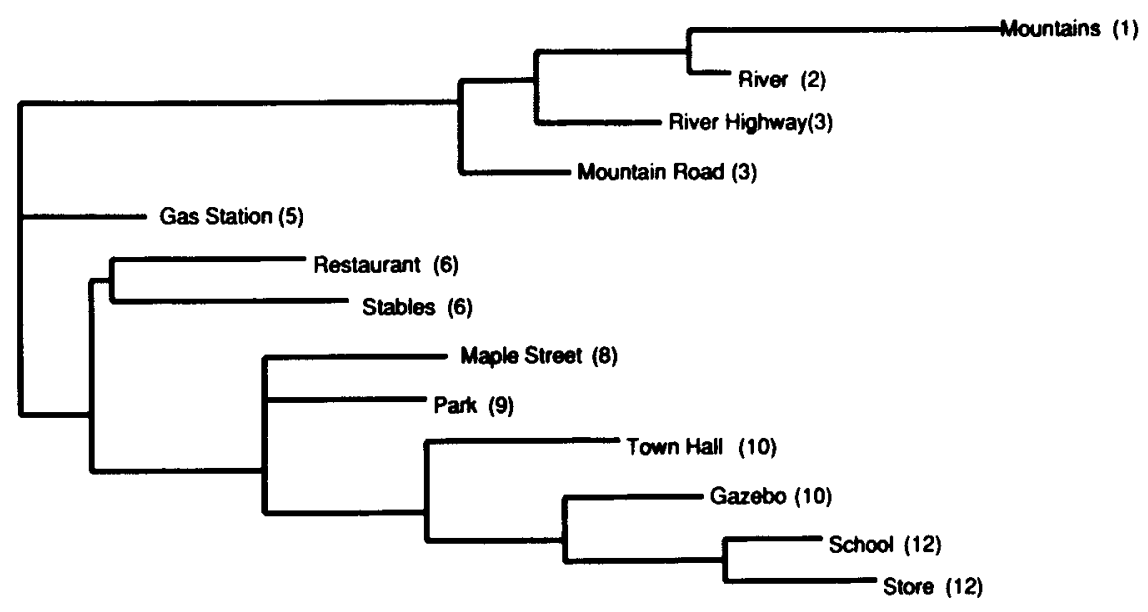

Figure 4. ADDTREE solution of description order for Town. Parenthetical numbers indicate median order of recall. 


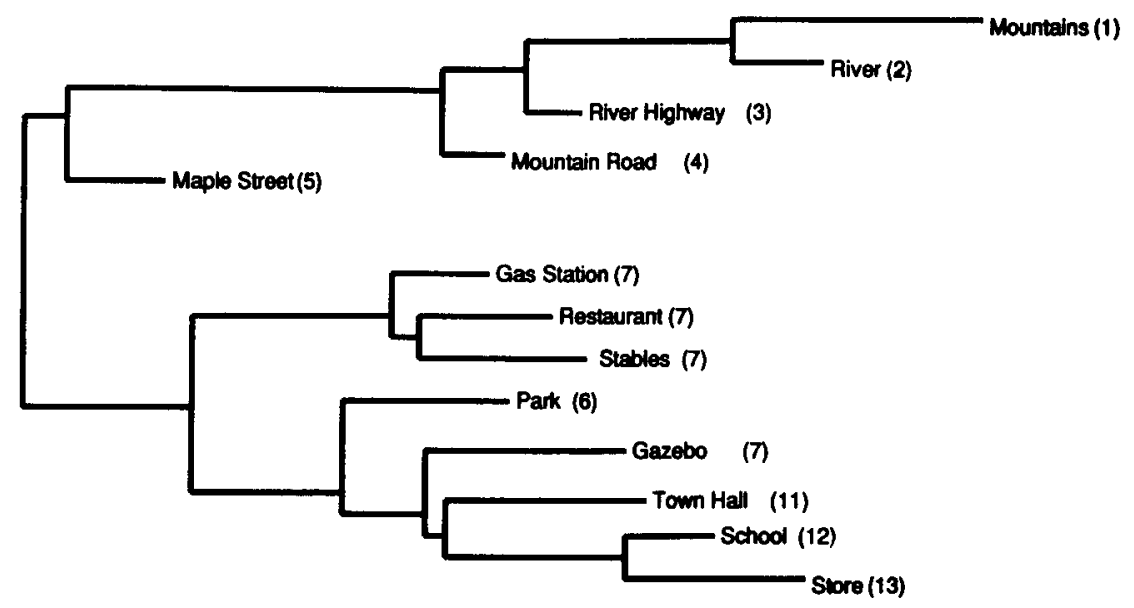

Figure 5. ADDTREE solution of drawing order for Town. Parenthetical numbers indicate median order of recall.

tent with the concordance of recall order between maps and descriptions, both across and between subjects, and will be examined in more detail in the next section.

Specific features of orders. In addition to the ADDTREE analysis, we examined specific features of the drawing and description orders, including starting point, clusters, hierarchical structures, and direction of tour. The groupings for the hierarchical structures corresponded to the major clusters in the ADDTREE solutions. Given the different features of each environment, these microanalyses depended on the individual environment. Because these analyses did not require equal numbers of landmarks between and within tasks, the data eliminated from the overall ordering analyses were again included.

For the Town, we looked at the starting point, the location of the first mentioned road, the location of the first mentioned details, and the complete or partial use of the hierarchy established by the size of the environment's features. The results of these analyses for both map-drawing orders and description orders are shown in Table 3. The predominant starting point was the White Mountains, the northern border. The only other starting points were

Amusement Park: Description Order

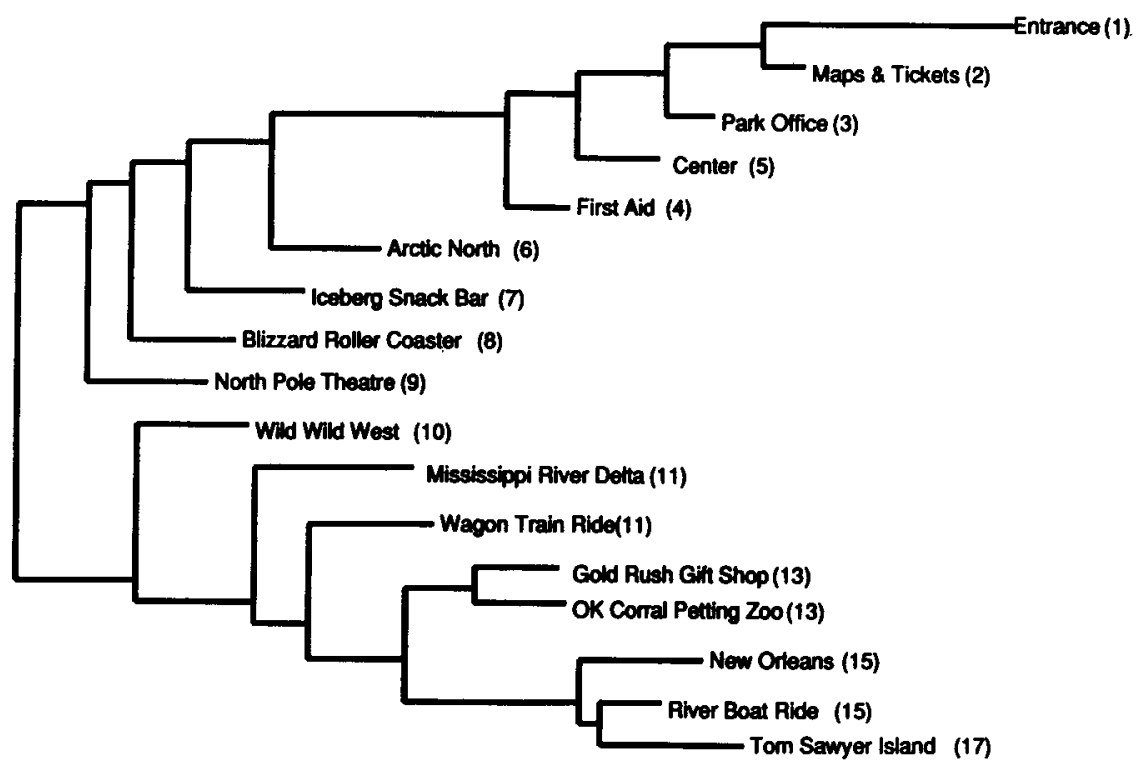

Figure 6. ADDTREE solution of description order for Amusement Park. Parenthetical numbers indicate median order of recall. 


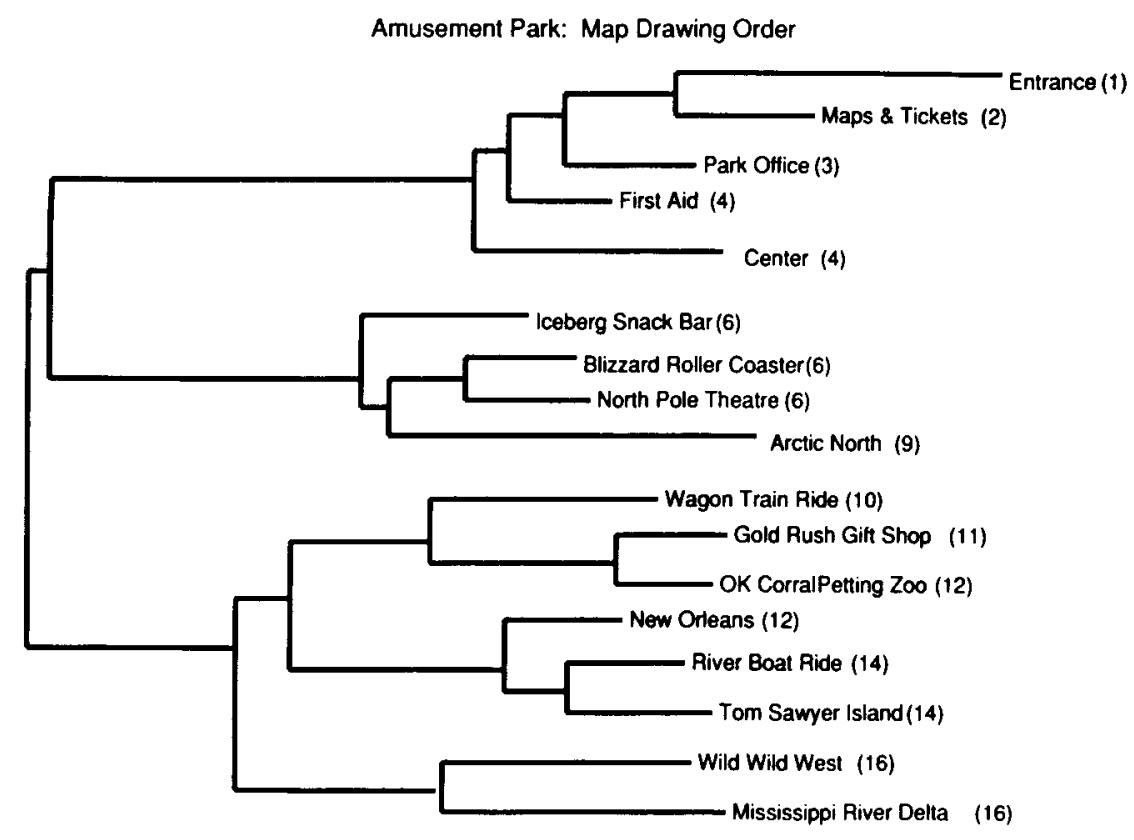

Figure 7. ADDTREE solution of drawing order for Amusement Park. Parenthetical numbers indicate median order of recall.

the major roads: River Highway and Mountain Road. The White Mountains, the largest feature of the environment, were used significantly more often as a starting point both for maps $\left[\chi^{2}(1, N=22)=8.84, p<.01\right]$ and for descriptions $\left[\chi^{2}(1, N=22)=4.96, p<.05\right]$. The first road mentioned was more often the River Highway along the southern border than the Mountain Road along the eastern border. The first details mentioned corresponded to the first road mentioned. The details along the River Highway in the South were usually mentioned before the details along the Mountain Road in the East. Despite the similarity of trends for both maps and descriptions, neither effect, first road or first detail, was significant. The Town was created from landmarks of three magnitudes: large natural features such as the mountains and the river, major highways, and individual buildings. The subjects recalled landmarks in decreasing order of size in both tasks. The use of the size hierarchy was significantly above chance for the maps $\left[\chi^{2}(2, N=22)=19.21, p<.01\right]$, but not for the descriptions.

For the Amusement Park, the interesting properties of the orders included the starting point, the first region men-

Convention Center: Description Order

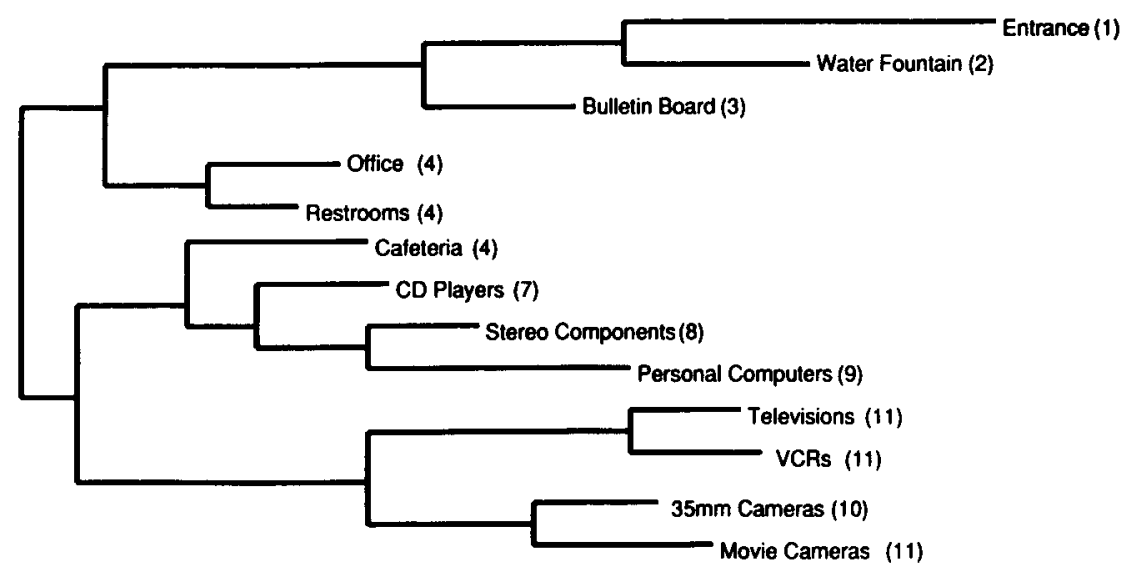

Figure 8. ADDTREE solution of description order for Convention Center. Parenthetical numbers indicate median order of recall. 
Convention Center: Map Drawing Order

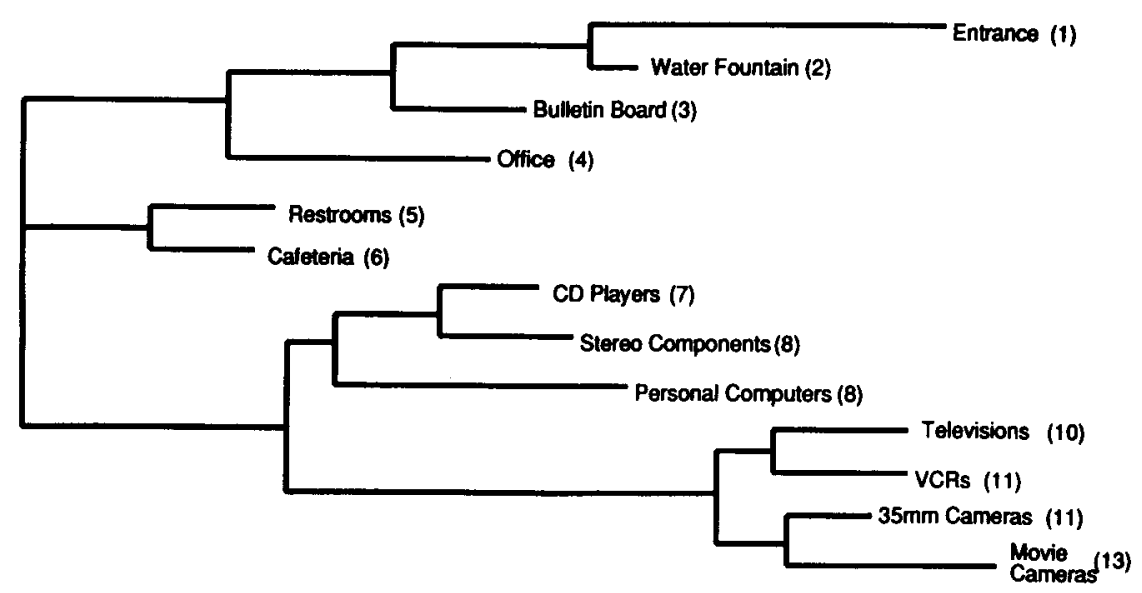

Figure 9. ADDTREE solution of drawing order for Convention Center. Parenthetical numbers indicate median order of recall.

tioned after starting, the first southern region mentioned, and the type of hierarchy, if any, used. Table 4 shows these results. Two main starting points emerged, the entrance to the park and the northernmost region, the Arctic North. Of these two starting points, the entrance was used marginally significantly more often than the northern region for the maps $\left[\chi^{2}(1, N=22)=2.74, p<.1\right]$ and significantly more often for the descriptions $\left[\chi^{2}(1, N=22)=11.98, p<.01\right]$. Regardless of starting point, the subjects significantly more often mentioned the items in the northern part of the environment before those in the South both for maps $\left[\chi^{2}(1, N=22)=5.38\right.$, $p<.05]$ and for descriptions $\left[\chi^{2}(1, N=22)=3.92\right.$, $p<.05]$. That is, the subjects tended to proceed from North to South, or top to bottom. There were two southern sections, an eastern one and a western one. The subjects proceeded significantly more often from West to East, or left to right, in the descriptions $\left[\chi^{2}(1, N=22)=11.98\right.$, $p<.01]$. The trend was in the same direction for maps, but it did not reach significance. Finally, the subjects could proceed hierarchically by first mentioning the three major subareas (breadth first) or by first describing one subarea entirely, then another, and then the third (depth first). The subjects used one of these hierarchies significantly more than none at all both for maps $\left[\chi^{2}(1, N=22)=11.46\right.$, $p<.01]$ and for descriptions $\left[\chi^{2}(1, N=22)=11.46\right.$, $p<.01]$. The subjects used breadth-first hierarchies more often than depth-first hierarchies on both tasks, but this did not reach significance for either task.

For the Convention Center, we examined starting point, dominant direction of tour, and ordering of environment by defined segments. Table 5 shows these results for map and description orders. The agreement about where to start either task was the most striking. As they did with the Amusement Park, the subjects tended to use the entrance as a starting point both for the maps $\left[\chi^{2}(1, N=23)\right.$ $=7.17, p<.05]$ and for the descriptions $\left[\chi^{2}(1, N=23)\right.$ $=11.98, p<.01]$. Primary direction of tour mainly indicated what was not preferred. The subjects chose a counterclockwise direction significantly less often than either a clockwise direction or a pattern following the walls of the building, but noncontiguously, both for the maps $\left[\chi^{2}(1, N=23)=11.98, p<.01\right]$ and for the descriptions $\left[\chi^{2}(1, N=23)=3.92, p<.05\right]$ The subjects showed little preference between the consistent clockwise direction and the noncontiguous order. The Convention Center consisted of an outer ring and an inner block. The subjects could first describe the outer ring, or first de-

Table 3

Town: Ordering Landmarks

\begin{tabular}{lrr}
\hline & \multicolumn{2}{c}{ Task } \\
\cline { 2 - 3 } & Map & Description \\
\hline Starting Point & & \\
$\quad$ Mountains & $91 \%$ & $82 \%$ \\
$\quad$ River Highway & $9 \%$ & $14 \%$ \\
$\quad$ Mountain Road & $0 \%$ & $5 \%$ \\
$\quad$ First Road & & \\
$\quad$ River Highway & $73 \%$ & $59 \%$ \\
$\quad$ Mountain Road & $27 \%$ & $41 \%$ \\
First Details & & \\
$\quad$ On River Highway & $59 \%$ & $64 \%$ \\
$\quad$ On Mountain Road & $41 \%$ & $36 \%$ \\
Hierarchy & & \\
$\quad$ Border-roads-details & $96 \%$ & $59 \%$ \\
2 of 3 ordered & $4 \%$ & $14 \%$ \\
2 of 3 not ordered & $0 \%$ & $27 \%$ \\
\hline
\end{tabular}

Note-Entries are percentages of subjects. 
Table 4

Amusement Park: Ordering Landmarks

\begin{tabular}{lcc}
\hline & \multicolumn{2}{c}{ Task } \\
\cline { 2 - 3 } & Map & Description \\
\hline Starting Point & & \\
Entrance & $68 \%$ & $77 \%$ \\
Northern section & $27 \%$ & $23 \%$ \\
Southern section & $5 \%$ & $0 \%$ \\
First Direction & & \\
North & $59 \%$ & $86 \%$ \\
South & $41 \%$ & $14 \%$ \\
First South & & \\
West & $59 \%$ & $73 \%$ \\
East & $32 \%$ & $27 \%$ \\
Both & $9 \%$ & $0 \%$ \\
Hierarchy & & \\
$\quad$ Depth first & $59 \%$ & $59 \%$ \\
Breadth first & $36 \%$ & $5 \%$ \\
Neither & $5 \%$ & \\
\hline Note- Entries are percentages of &
\end{tabular}

Note-Entries are percentages of subjects.

Table 5

Convention Center: Ordering Landmarks

\begin{tabular}{lcc}
\hline & \multicolumn{2}{c}{ Task } \\
\cline { 2 - 3 } & Map & Description \\
\hline Starting Point & & \\
$\quad$ Entrance & $87 \%$ & $96 \%$ \\
Other & $13 \%$ & $4 \%$ \\
Dominant Direction & & \\
$\quad$ Clockwise & $35 \%$ & $65 \%$ \\
Counterclockwise & $4 \%$ & $22 \%$ \\
None & $61 \%$ & $13 \%$ \\
Section Order & & \\
Outside first & $48 \%$ & $48 \%$ \\
Both & $36 \%$ & $48 \%$ \\
Inside first & $17 \%$ & $4 \%$ \\
\hline
\end{tabular}

Note-Entries are percentages of subjects.

scribe the inner core, or go back and forth between the outer ring and the inner core. The subjects avoided beginning with the inner block, preferring either to begin with the outer ring or to mix the two segments both for the maps $\left[\chi^{2}(1, N=23)=5.38, p<.05\right]$ and for the descriptions $\left[\chi^{2}(1, N=23)=11.98, p<.01\right]$. Approximately equal numbers of subjects used the other alternatives.

\section{DISCUSSION}

Different response measures showed effects of expected task and actual task, but the most dramatic effects were for the specific environments. Memory for the maps was excellent. Most subjects included all or almost all the landmarks in their drawings and descriptions. Memory was slightly but significantly better for depictions than for descriptions. Two possible explanations for the superiority of maps over descriptions come to mind. First, it is easier to add a landmark remembered later to a drawing than to a description. A drawing will usually have room for a missing landmark. Next, a map drawing provides a better cue to a missing map element than does a description as it is closer to the original stimulus and there are visual/spatial cues pointing to its absence. Task expectations, to draw or to describe, had no effects on overall memory, nor did order of performing the two tasks.

There were three sets of measures of organization, the correspondence measures (concordances and correlations), the trees, and the tables, all based on the order of landmarks in depictions and descriptions. The two correspondence measures showed considerable agreement of recall order both within and across subjects and within and across tasks. These correspondences, while high and significant, were not perfect, probably because of the existence of two popular and only partly correlated orders for most environments. Although the first task performed did not have any apparent effect on the coefficients of concordance, task expectations did. For each of the three environments, coefficients were higher when the subjects expected to describe the environment than when they expected to draw it. This finding, a trend that did not reach significance, suggests that the expectation to describe induced the subjects to impose more order on the elements of the environment.

The trees provided a fine-grained analysis of the organization, revealing more clustering in maps and more chaining in descriptions. The content of the larger clusters was similar for descriptions and depictions of the same environment, but within those clusters, order of landmarks was more consistent in descriptions than in drawings. That is consistent with the finding of higher concordances when the subjects expected to describe the environments. This makes sense in light of the communicative requirements of the two media. A description is serial in that only one topic can be mentioned at a time, yet it should be coherent throughout. One way of assuring coherence is by establishing links between topics, in this case, landmarks. The order in which landmarks are drawn is not part of the communicative act intended with a drawing. The completed drawing itself provides all the information. Thus, communicative considerations suggest that the orders of descriptions should be tightly constrained, as in fact they were. More surprising is the fact that there was considerable agreement across subjects as to what those orders should be, and that the same global clusters appeared in the drawings and the descriptions.

The tables examined the ordering between and, to some extent, within the larger clusters revealed by the trees. This analysis uncovered many of the commonalities of organization between descriptions and depictions. Because the maps were not designed to test hypotheses about organization and the principles of organization were discovered after the fact, some of the conclusions are somewhat speculative. Overall organization appeared to depend on both spatial and functional aspects of the environments (see van Sommers, 1984, for corroborative data from other kinds of pictures). Spatial organization seemed to be of three types: that based on proximity, that based on 
scale, and that based on direction conventions. Spatial proximity, spatial scale, and functional features were especially apparent in establishing the subgroups of the hierarchical organization and the conventional directions apparent in the linking of subgroups. Although there have been claims that most spatial descriptions take readers on a mental tour (Levelt, 1982; Linde \& Labov, 1975), of the present descriptions, many took a survey perspective on the environments, others took a mental tour, and still others mixed the two perspectives (Taylor \& Tversky, 1990).

Hierarchical organization was generally used for the Town and the Amusement Park and less frequently used for the Convention Center. By hierarchical organization, here we mean the reliable tendency of subjects to subdivide the environments and to draw or describe one set of features prior to another. For the Town, the hierarchy was based on scale: the larger, global features compared with the smaller, local ones. Most subjects, especially in drawing, began with the large border features, continued with the major roads, and then placed the smallest features, the buildings. In students' descriptions of their dorm rooms, Shanon (1984) found hierarchical organization based on size, as did Ehrich and Koster (1983) in descriptions of dollhouse rooms. For the Amusement Park, the hierarchy was based on a combination of spatial proximity and semantic theme; these were correlated, as they often are in natural environments. Most subjects, whether drawing or describing, explored the hierarchy depth first, that is, completing one region before going on to the next. A sizable minority of subjects took a breadth-first approach, that is, giving an overview of the regions before giving the details of each. For the Convention Center, the subjects in each medium were about evenly divided between those who proceeded hierarchically and those who did not. The hierarchy in this environment was based on groupings of landmarks that were all the same scale. Those who proceeded hierarchically tended to first describe or depict the outer corridor, the larger of the corridors, and then the inner corridor. Those who did not proceed hierarchically tended to describe outer and inner corridors alternately, circling once through the exhibits.

Yet another basis for hierarchical organization was functional. Two of the environments had entrances. The entrance to the Amusement Park was located to the left and toward the top, a conventionally preferred starting point, but that of the Convention Center was located in the lower right, opposite to the convention of starting in the top left. Irrespective of location, the entrance was used as a starting point in nearly all descriptions and depictions for both the Amusement Park and the Convention Center. Previous research, some of which was discussed earlier, has also demonstrated hierarchical organization in mental representations of spatial environments. The present research has gone beyond the demonstration of hierarchical organization by illuminating some of the sources of the hierarchies, both of the division into groups and of the linking of groups.
Where there was no spatial or functional basis for linking groups or clusters, linking appeared to be based on conventional orders. Reading order, that is, top-down, left-right, is a preferred order in drawing and perceptual search tasks, even those that are wordless (Goodnow, 1977; Kugelmass \& Lieblich, 1979; Tversky et al., 1991). That preference may be related to preferences in language for first expressing upward, and then downward, concepts, as in "above-below," "top-bottom," and "highlow," and for first expressing vertical and then horizontal dimensions, as in "height and width." These preferences may originate in perception (see especially Clark, 1973, but see also Cooper \& Ross, 1975, and Franklin \& Tversky, 1990). Reading order was evident in the Amusement Park, where descriptions and depictions typically proceeded first up (North), ${ }^{1}$ then down and left (West), and then down and right (East). Note that this is despite the fact that in English we say "East-West" rather than "West-East." The dominance of vertical over horizontal was also evident in the recall of the Town. Of the two large, bordering landmarks, the top (North) mountain range was selected prior to the left (West) river. In a similar task, Denis and Denhière (1990) found that descriptions frequently proceeded top-down and left-right. Another common conventional order is clockwise. Only the Convention Center offered an opportunity to check preferences for circular direction. There, for those using a circular order, more subjects chose to go clockwise rather than counterclockwise.

On the face of it, drawing and descriptions appear to be very different kinds of communications. Thus the similarity of the global organizations of the descriptions and depictions seems all the more remarkable. The commonality could be due to the way the environments were organized or to underlying communicative goals. We argue that these two factors are intertwined. Communicators presumably want their audiences to comprehend and remember what is described or depicted. It is well known that grouping, hierarchical organization, and coherent linking contribute to comprehensibility and memorability, so it is reasonable to expect communicators to use these devices to make the task of those receiving the messages easier. Levelt (1982) and Robin and Denis (1991) found that senders order discourse to reduce the memory load of receivers.

On the surface, these considerations may not seem as applicable to depictions, which communicate as completed wholes, as to descriptions. Both tasks, however, require communication within the same person, the subject, who needs first to comprehend and then to remember the map to be drawn or described. As Brown and Lenneberg (1954) intimated, memory is in some sense a social act, of communicating with future versions of ourselves. The same organization processes that facilitate comprehension facilitate memory and communication, within the same person or between people.

Yet another communicative link underlies the similarity of organization in depictions and descriptions of the 
same environments. Language is a surrogate for experience. Just as the goal of the drawing was to recreate a picture, an actual one, so the goal of the description was to recreate a picture, in this case, in the mind of another. That descriptions can create mental pictures highly similar to their sources was demonstrated in the mirror image of the present study. In that work (Taylor \& Tversky, 1992), the subjects read descriptions of environments such as the present ones and later drew maps of them. The descriptions the subjects read were quite similar to those that the subjects in the present experiment wrote. And the maps that they constructed solely from descriptions were quite similar to the maps the subjects in the present experiment studied. Given the similarity of goals, of creating pictures, real or mental, the similarity of organization of depictions and descriptions seems natural.

\section{REFERENCES}

Bons, F. (1955). Primitive art. New York: Dover. (Original work published 1927)

Brown, L. A. (1977). The story of maps. New York: Dover. (Original work published 1949)

Brown, R., Lenneberg, E. H. (1954). A study in language and cognition. Journal of Abnormal \& Social Psychology, 49, 454-462.

Chase, W. G., \& ChI, M. T. H. (1981). Cognitive skill: Implications for spatial skill in large-scale environments. In J. H. Harvey (Ed.), Cognition, social behavior, and the environment. Hillsdale, NJ: Erlbaum.

Clark, H. H. (1973). Space, time, semantics, and the child. In T. E. Moore (Ed.), Cognitive development and the acquisition of language (pp. 27-63). New York: Academic Press.

COOPER, W. E., Ross, J. R. (1975). World order. In R. E. Grossman, L. J. San, \& T. J. Vance (Eds.), Papers from the parasession on functionalism (pp. 63-112). Chicago: Chicago Linguistic Society.

Denis, M., \& DenhiEkR, G. (1990). Comprehension and recall of spatial descriptions. European Bulletin of Cognitive Psychology, 10, 115-143.

EHRich, V., \& Koster, C. (1983). Discourse organization and sentence form: The structure of room descriptions in Dutch. Discourse Processes, 6, 169-195.

FrankLin, N., \& Tversky, B. (1990). Searching imagined environments. Journal of Experimental Psychology: General, 119, 63-76.

GeLB, I. J. (1963). A study of writing. Chicago: University of Chicago Press.

GoodNow, J. (1977). Children's drawing. London: Open Books.

HAGEN, M. A. (1986). Varieties of realism: Geometries of representational art. Cambridge: Cambridge University Press.

HiRTLE, S. C., JoNiDES, J. (1985). Evidence of hierarchies in cognitive maps. Memory \& Cognition, 13, 208-217.

Kugelmass, S., \& Lieblich, A. (1979). The impact of learning to read on directionality in perception: A further cross-cultural analysis. Human Development, 22, 406-415.

LEVELT, W. J. M. (1982). Linearization in describing spatial networks. In S. Peters \& E. Saarinen (Eds.), Processes, beliefs, and questions (pp. 199-220). Dordrecht, The Netherlands: Reidel.

LINDE, C., LABOV, W. (1975). Spatial networks as a site for the study of language and thought. Language, 51, 924-939.

MCNAMARA, T. P. (1986). Mental representations of spatial relations. Cognitive Psychology, 18, 87-121.

NoBlE, J. N. (1981). The mapmakers. New York: Vintage.

RoBIN, F., DENIS, M. (1991). Description of perceived or imagined spatial networks. In R. H. Logie \& M. Denis (Eds.), Mental images in human cognition (pp. 141-152). Amsterdam: North-Holland.

Sattath, S., Tversky, A. (1977). Additive similarity trees. Psychometrika, 42, 319-345.

Shanon, B. (1984). Room descriptions. Discourse Processes, 6, 319-352.

Southworth, M., \& SOUThWORTh, S. (1982). Maps: A visual survey and design guide. Boston: Little, Brown.

STEVENS, A., \& CouPE, P. (1978). Distortions in judged spatial relations. Cognitive Psychology, 10, 422-437.

TAYLOR, H. A., TVERSKy, B. (1990, November). Spatial descriptions and depictions. Paper presented at the meeting of the Psychonomic Society, New Orleans.

TAYLOR, H. A., TVERSKY, B. (1992). Spatial mental models derived from survey and route descriptions. Joumal of Memory \& Language, 31, 261-282.

TULVING, E. (1962). Subjective organization in free recall of "unrelated" words. Psychological Review, 69, 344-354.

TVERSKY, B. (1973). Encoding processes in recognition and recall. Cognitive Psychology, 5, 275-287.

TVERSKY, B. (1981). Distortions in memory for maps. Cognitive Psychology, 13, 407-433.

TVersky, B. (1992). Distortions in cognitive maps. Geoforum, 23, 131-138.

Tversky, B., Kugelmass, S., Winter, A. (1991). Cross-cultural and developmental trends in graphic productions. Cognitive Psychology, 23, 515-557.

TVersKY, B., SChiano, D. (1989). Perceptual and conceptual factors in distortions in memory for maps and graphs. Joumal of Experimental Psychology: General, 118, 387-398.

van Sommers, P. (1984). Drawing and cognition: Descriptive and experimental studies of graphic production processes. Cambridge: Cambridge University Press.

\section{NOTE}

1. There is a confounding here with branch size. The North branch is smaller than the South branch, which has two subgroups. Levelt (1982) and Robin and Denis (1991) found that subjects tended to describe a short branch before a long one. However, in the case of the Convention Center, the subjects who circled twice chose the longer branch before the shorter branch. This seems to be a case of natural order considerations superseding those of memory load.

\section{APPENDIX}

\section{Example of Town Description}

The map is of a township with a range of $\mathrm{mtns}$. to the $\mathrm{N}$ and a river running along the $\mathrm{W}$ edge. There was no scale on the map, but both geographical features look to be a fair distance from the town. Two main roads intersect for the town's turnoff: River Rd. which runs E-W on the S side of town and across the river and Mtn. Rd. which runs S out of the mtns. towards the town, and finally intersects with River Rd. The town itself has kind of a main square or cul-de-sac off the W side of Mtn. Rd. just above the River Rd./Mtn. Rd. junction. In the center is a gazebo. $\mathrm{E}$ of the gazebo, across Mtn. Rd., is the town hall, to the $\mathrm{N}$ across the cul-de-sac is the store and to the $\mathrm{W}$ across the cul-de-sac is the school. Three buildings stand near the junction of Mtn. and River Rds.: a restaurant on the N-E corner, stables on the S side (a bit to W of the junction) and a gas station across from the stables on the $\mathrm{N}-\mathrm{W}$ corner of the junction. 


\section{Example of Amusement Park Description}

This park (has no name) has 2 entrances which fork together as you go east. The park entrance is where you buy tickets and maps and this leads out to a big round open space from which 3 main paths diverge, 2 south, one north. In this space there is the park office and the first aid in separate buildings. Going to the left (north) will take you to the North Pole area as you go up the path, to your left is the North Pole arena, and to the right is first a snack bar with ice-cold popsicles and also a grill. Beyond this is a big roller coaster ride with lots of space to wait in line. If you didn't want to go to the North Pole place, you could turn right after you get into the park. A sharp right will take you to the Wild, Wild West, where you can either take a plain path or wagon ride to get to the Children's OK Corral with harmless animals for children to pet, and also t-shirts, amusements, and photos. The last big attraction is the Mississippi River Delta, which has Tom Sawyer's Island (with Lost Cave and Becky's Pond) and is accessible by a River Boat, which goes by a fallen tree. Or a path will take you down Bourbon Street, where you could go to hear jazz or eat at the French cafe. The Mississippi River Delta is located South as you walk in the entrance (or south-south-east, to the left of the Wild, Wild West).

\section{Example of Convention Center Description}

The entrance is on the east side of the building. As you enter, there is a water fountain on your left, and beyond it a bulletin board. As you walk down the corridor in front of you, you pass movie cameras on your right, then $35 \mathrm{~mm}$ cameras. On your left is the office. As you reach the end of the corridor, the restrooms are directly ahead of you, side by side. Turn right and continue walking; the cafeteria will be on your left. Turn right again at the end of the corridor; the CD players will be just ahead on your left, and the televisions on your right. Farther up, the VCRs are on the right and stereo components on your left. Turn right at the end of the hallway; you will pass personal computers on your left and then find yourself back at the entrance.

(Manuscript received August 15, 1991; revision accepted for publication March 17, 1992.) 